\title{
Front Matter: Volume 7274
}

, "Front Matter: Volume 7274," Proc. SPIE 7274, Optical Microlithography XXII, 727401 (8 April 2009); doi: 10.1117/12.828059

SPIE. Event: SPIE Advanced Lithography, 2009, San Jose, California, United States 


\title{
PROCEEDINGS OF SPIE
}

\section{Optical Microlithography XXII}

\author{
Harry J. Levinson \\ Mircea V. Dusa \\ Editors
}

24-27 February 2009

San Jose, California, United States

Sponsored by

SPIE

Cooperating Organization

SEMATECH Inc. (United States)

Published by

SPIE 
The papers included in this volume were part of the technical conference cited on the cover and title page. Papers were selected and subject to review by the editors and conference program committee. Some conference presentations may not be available for publication. The papers published in these proceedings reflect the work and thoughts of the authors and are published herein as submitted. The publisher is not responsible for the validity of the information or for any outcomes resulting from reliance thereon.

Please use the following format to cite material from this book:

Author(s), "Title of Paper," in Optical Microlithography XXII, edited by Harry J. Levinson, Mircea V. Dusa, Proceedings of SPIE Vol. 7274 (SPIE, Bellingham, WA, 2009) Article CID Number.

ISSN 0277-786X

ISBN 9780819475275

Published by

SPIE

P.O. Box 10, Bellingham, Washington 98227-0010 USA

Telephone +1 3606763290 (Pacific Time) · Fax +1 3606471445

SPIE.org

Copyright (c) 2009, Society of Photo-Optical Instrumentation Engineers

Copying of material in this book for internal or personal use, or for the internal or personal use of specific clients, beyond the fair use provisions granted by the U.S. Copyright Law is authorized by SPIE subject to payment of copying fees. The Transactional Reporting Service base fee for this volume is $\$ 18.00$ per article (or portion thereof), which should be paid directly to the Copyright Clearance Center (CCC), 222 Rosewood Drive, Danvers, MA 01923. Payment may also be made electronically through CCC Online at copyright.com. Other copying for republication, resale, advertising or promotion, or any form of systematic or multiple reproduction of any material in this book is prohibited except with permission in writing from the publisher. The CCC fee code is 0277-786X/09/\$18.00.

Printed in the United States of America.

Publication of record for individual papers is online in the SPIE Digital Library.

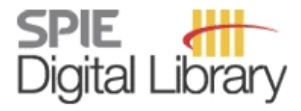

SPIEDigitalLibrary.org

Paper Numbering: Proceedings of SPIE follow an e-First publication model, with papers published first online and then in print and on CD-ROM. Papers are published as they are submitted and meet publication criteria. A unique, consistent, permanent citation identifier (CID) number is assigned to each article at the time of the first publication. Utilization of CIDs allows articles to be fully citable as soon they are published online, and connects the same identifier to all online, print, and electronic versions of the publication. SPIE uses a six-digit CID article numbering system in which:

- The first four digits correspond to the SPIE volume number.

- The last two digits indicate publication order within the volume using a Base 36 numbering system employing both numerals and letters. These two-number sets start with 00, 01, 02, 03, 04 , $05,06,07,08,09,0 A, 0 B \ldots 0 Z$, followed by 10-1Z, 20-2Z, etc.

The CID number appears on each page of the manuscript. The complete citation is used on the first page, and an abbreviated version on subsequent pages. Numbers in the index correspond to the last two digits of the six-digit CID number. 


\section{Contents}

\section{Part One}

xvii Symposium Committees

xix Conference Committee

\section{SESSION 1 INVITED SESSION}

727402 Alternative optical technologies: more than curiosities? (Keynote Paper) [7274-01]

B. W. Smith, Rochester Institute of Technology (United States)

\section{SESSION 2 RESOLUTION ENHANCEMENT}

727404 Overcoming the challenges of $22-\mathbf{n m}$ node patterning through litho-design co-optimization [7274-03]

M. Burkhardt, J. C. Arnold, IBM Research (United States); Z. Baum, IBM SRDC (United States); S. Burns, J. Chang, J. Chen, IBM Research (United States); J. Cho, V. Dai, Y. Deng, Advanced Micro Devices, Inc. (United States); S. Halle, G. Han, S. Holmes, D. Horak, IBM SRDC (United States); S. Kanakasabapathy, IBM Research (United States); R. H. Kim, Advanced Micro Devices, Inc. (United States); A. Klatchko, Freescale Corp. (United States); C. S. Koay, A. Krasnoperova, IBM SRDC (United States); Y. Ma, Advanced Micro Devices, Inc. (United States); E. McLellan, K. Petrillo, S. Schmitz, IBM SRDC (United States); C. Tabery, Advanced Micro Devices, Inc. (United States); Y. Yin, L. Zhuang, IBM SRDC (United States); Y. Zou, J. Kye, Advanced Micro Devices, Inc. (United States); V. Paruchuri, IBM Research (United States); S. Mansfield, IBM SRDC (United States); C. Spence, Advanced Micro Devices, Inc. (United States); M. Colburn, IBM Research (United States)

727405 Improved model predictability by machine data in computational lithography and application to laser bandwidth tuning [7274-04]

S. Hunsche, Q. Zhao, X. Xie, Brion Technologies Inc., ASML (United States); R. Socha, ASML (United States); H.-Y. Liu, Brion Technologies (United States); P. Nikolsky, A. Ngai, P. van Adrichem, ASML Netherlands B.V. (Netherlands); M. Crouse, ASML Corp. (United States); I. Lalovic, Cymer, Inc. (United States)

727407 Contact mask optimization and SRAF design [7274-06]

U. P. Schroeder, Infineon Technologies (United States) and IBM Semiconductor Research and Development Ctr. (USA); C. Tabery, Advanced Micro Devices Inc. (United States) and IBM Semiconductor Research and Development Ctr. (USA); B. Morgenfeld, IBM (United States) and IBM Semiconductor Research and Development Ctr. (USA); H. Kanai, Toshiba Corp. (United States) and IBM Semiconductor Research and Development Ctr. (USA) 


\section{SESSION 3 SOURCE AND MASK OPTIMIZATION}

727408 A study of source and mask optimization for ArF scanners [7274-07]

T. Matsuyama, T. Nakashima, T. Noda, Nikon Corp. (Japan)

727409 Intensive optimization of masks and sources for 22nm lithography [7274-08]

A. E. Rosenbluth, D. O. Melville, IBM Thomas J. Watson Research Ctr. (United States); K. Tian, S. Bagheri, J. Tirapu-Azpiroz, K. Lai, IBM Semiconductor Research and Development Ctr. (United States); A. Waechter, IBM Thomas J. Watson Research Ctr. (United States); T. Inove, IBM Tokyo Research Lab. (Japan); L. Ladanyi, F. Barahona, K. Scheinberg, IBM Thomas J. Watson Research Ctr. (United States); M. Sakamoto, H. Muta, IBM Tokyo Research Lab. (Japan); E. Gallagher, T. Faure, M. Hibbs, IBM Systems and Technology Group (United States); A. Tritchkov, Y. Granik, Mentor Graphics Corp. (United States)

7274 OA Experimental result and simulation analysis for the use of pixelated illumination from source mask optimization for 22nm logic lithography process [7274-09]

K. Lai, IBM Microelectronics (United States); A. E. Rosenbluth, IBM Yorktown Research Ctr. (United States); S. Bagheri, IBM Microelectronics (United States); J. Hoffnagle, IBM Almaden Research Ctr. (United States); K. Tian, IBM Microelectronics (United States); D. Melville, IBM Yorktown Research Ctr. (United States); J. Tirapu-Azpiroz, IBM Microelectronics (United States); M. Fakhry, Mentor Graphics Corp. (United States); Y. Kim, IBM Almaden Research Ctr. (United States); S. Halle, G. McIntyre, IBM Microelectronics (United States); A. Wagner, IBM Yorktown Research Ctr. (United States); G. Burr, IBM Almaden Research Ctr. (United States); M. Burkhardt, IBM Yorktown Research Ctr. (United States); D. Corliss, IBM Microelectronics (United States); E. Gallagher, T. Faure, M. Hibbs, IBM Mask House (United States); D. Flagello, ASML US, Inc. (United States); J. Zimmermann, B. Kneer, F. Rohmund, F. Hartung, C. Hennerkes, M. Maul, Carl Zeiss SMT AG (Germany); R. Kazinczi, A. Engelen, R. Carpaij, R. Groenendijk, J. Hageman, ASML (Netherlands)

$7274 \mathrm{OB}$ Enabling process window improvement at $45 \mathrm{~nm}$ and $32 \mathrm{~nm}$ with free-form DOE illumination [7274-10]

T. H. Coskun, A. Sezginer, V. Kamat, M. Kruger, B. Yenikaya, Cadence Design Systems, Inc. (United States); J. Carriere, J. Stack, M. Himel, Tessera (United States)

7274 OC Benefits and trade-offs of global source optimization in optical lithography [7274-11] K. Tian, A. Krasnoperova, IBM Semiconductor Research and Development Ctr. (United States); D. Melville, A. E. Rosenbluth, D. Gil, IBM Thomas J. Watson Research Ctr. (United States); J. Tirapu-Azpiroz, K. Lai, S. Bagheri, C. Chen, B. Morgenfeld, IBM Semiconductor Research and Development Ctr. (United States)

\section{SESSION 4 SPACER-BASED PROCESSES}

7274 OD Demonstration of 32nm half-pitch electrical testable NAND FLASH patterns using self-aligned double patterning [7274-149]

S. Sun, C. Bencher, Y. Chen, H. Dai, M. Cai, J. Jin, P. Blanco, L. Miao, P. XU, X. XU, J. YU, R. Hung, S. Oemardani, O. Chan, C.-P. Chang, C. Ngai, Applied Materials, Inc. (United States) 
$7274 \mathrm{OE} \quad$ Advanced self-aligned double patterning development for sub-30-nm DRAM manufacturing [7274-12]

W. Shiu, H. J. Liu, J. S. Wu, T.-L. Tseng, C. T. Liao, C. M. Liao, J. Liu, T. Wang, Nanya Technology Corp. (Taiwan)

7274 OF Process step reduction at negative tone spacer patterning technique using developer soluble bottom ARC [7274-13]

W.-Y. Jung, J.-D. Eom, S.-M. Jeon, J.-H. Lee, B.-S. Lee, J.-W. Kim, Hynix Semiconductor, Inc. (Korea, Republic of)

7274 OG Gridded design rule scaling: taking the CPU toward the 16nm node [7274-14]

C. Bencher, H. Dai, Y. Chen, Applied Materials, Inc. (United States)

\section{SESSION 5 DOUBLE PATTERNING I}

$7274 \mathrm{OH} \quad$ Split, overlap/stitching, and process design for double patterning considering local reflectivity variation by using rigorous 3D wafer-topography/lithography simulation [7274-16]

I. Kamohara, Synopsys Japan (Japan); T. Schmoeller, Synopsys GmbH (Germany)

$7274 \mathrm{Ol} \quad$ Advances and challenges in dual-tone development process optimization [7274-17] C. Fonseca, M. Somervell, S. Scheer, W. Printz, Tokyo Electron America, Inc. (United States); K. Nafus, S. Hatakeyama, Y. Kuwahara, T. Niwa, Tokyo Electron Kyushu, Ltd. (Japan); S. Bernard, R. Gronheid, IMEC (Belgium)

$72740 \mathrm{~J} \quad$ New process proximity correction using neural network in spacer patterning technology [7274-18]

F. Nakajima, T. Kotani, S. Tanaka, M. Asano, S. Inove, Toshiba Corp. Semiconductor Co. (Japan)

7274 OK $\quad 32 \mathrm{~nm}$ and below logic patterning using optimized illumination and double patterning [7274-19]

M. C. Smayling, Tela Innovations, Inc. (United States); V. Axelrad, Sequoia Design Systems, Inc. (United States)

7274 OM Analysis of topography effects on lithographic performance in double patterning applications [7274-21]

J. Siebert, Synopsys, Inc. (Germany); P. Brooker, Synopsys, Inc. (United States); T. Schmoeller, T. Klimpel, Synopsys, Inc. (Germany)

$7274 \mathrm{ON} \quad$ Ultimate contact hole resolution using immersion lithography with line/space imaging [7274-23]

V. Truffert, J. Bekaert, F. Lazzarino, M. Maenhoudt, A. Miller, M. Moelants, IMEC vzw (Belgium); T. Wu, IMEC vzw (Belgium) and TSMC, Ltd. (Taiwan)

$727400 \quad$ Efficient simulation and optimization of wafer topographies in double patterning [7274-24] F. Shao, P. Evanschitzky, T. Fühner, A. Erdmann, Fraunhofer Institute of Integrated Systems and Device Technology (Germany) 
$7274 \mathrm{OP}$ Investigating the impact of topography on pitch splitting double patterning using rigorous physical simulation [7274-22]

S. A. Robertson, T. Graves, J. J. Biafore, M. D. Smith, KLA-Tencor (United States)

\section{SESSION 7 TOOLS RELATED PROCESS CONTROL I}

7274 OQ Scanner OPC signatures: automatic vendor-to-vendor OPE matching [7274-86]

S. P. Renwick, Nikon Precision, Inc. (United States)

7274 OR Dense lines created by spacer DPT scheme: process control by local dose adjustment using advanced scanner control [7274-26]

J. Finders, ASML (Netherlands); M. Dusa, ASML US, Inc. (United States); B. Vleeming,

T. Fliervoet, B. Hepp, H. Megens, R. Groenendijk, J. Quaedackers, E. Mos, C. Leewis,

F. Bornebroek, ASML (Netherlands); M. Maenhoudt, M. Leblans, T. Vandeweyer,

G. Murdoch, E. Altamirano Sanchez, IMEC vzw (Belgium)

7274 OS Focus and dose characterization of immersion photoclusters [7274-27]

T. A. Brunner, D. Corliss, T. Wiltshire, C. P. Ausschnitt, IBM SRDC (United States)

7274 OT Model-based scanner tuning in a manufacturing environment [7274-114]

C. Y. Shih, R. C. Peng, T. C. Chien, Y. W. Guo, J. Y. Lee, C. L. Chang, P. C. Huang, H. H. Liu,

H. J. Lee, J. Lin, K. W. Chang, C. P. Yeh, Taiwan Semiconductor Manufacturing Co. (Taiwan); W. J. Shao, H. Cao, A. Bruguier, X. Xie, C. H. Chang, R. Aldana, Y. Cao, R. Goossens, Brion Technologies Inc., ASML(United States); S. Hsieh, ASML (Netherlands)

7274 OU Scanner-dependent optical proximity effects [7274-29]

J. K. Tyminski, Nikon Precision, Inc. (United States); T. Matsuyama, T. Nakashima, R. Inove,

Nikon Corp. (Japan)

\section{SESSION 8 TOOLS RELATED PROCESS CONTROL II}

7274 OV Challenges and solutions in the calibration of projection lens pupil-image metrology tools [7274-30]

S. Slonaker, B. Riffel, Nikon Precision, Inc. (United States); H. Nishinaga, Nikon Corp. (Japan)

$7274 \mathrm{OW} \quad$ Impact of CD and overlay errors on double-patterning processes [7274-31]

C. Lapeyre, S. Barnola, I. Servin, S. Gaugirana, CEA/LETI-Minatec (France); V. Salvetat, Nikon Precision Europe GmbH (France); N. Magome, A. J. Hazelton, Nikon Corp. (Japan); M. McCallum, Nikon Precision Europe GmbH (United Kingdom)

7274 0X Achieving overlay budgets for double patterning [7274-32]

A. J. Hazelton, N. Magome, S. Wakamoto, A. Tokui, Nikon Corp. (Japan); C. Lapeyre, S. Barnola, G. Jullien, CEA/LETI-Minatec (France); V. Salvetat, Nikon Precision Europe GmbH (Germany)

7274 OY Innovative pattern matching method considering process margin and scanner design information [7274-33]

K. Tsujita, K. Mikami, H. Ishii, R. Nakayama, M. Arakawa, T. Ueno, S. Fujie, K. Takahashi, Canon, Inc. (Japan) 
$7274 \mathrm{OZ} \quad$ A new method for post-etch OPC modeling to compensate for underlayer effects from integrated wafers [7274-34]

C. Sarma, Infineon Technologies NA (United States); A. Abdo, D. Dunn, D. Fischer, IBM Microelectronics (United States); K. Herold, Infineon Technologies NA (United States);

S. Mansfield, L. Tsou, IBM Microelectronics (United States)

727410 Optimal setting strategy for kernel-based OPC simulation engines [7274-35]

K. Kodera, S. Tanaka, T. Kotani, S. Inove, Toshiba Corp. Semiconductor Co. (Japan)

727411 Improving yield through the application of process window OPC [7274-36]

J. Tirapu Azpiroz, A. Krasnoperova, S. Siddiqui, K. Settlemyer, I. Graur, I. Stobert,

J. M. Oberschmidt, IBM Microelectronics (United States)

727412 Etch aware optical proximity correction: a first step toward integrated pattern engineering [7274-37]

D. N. Dunn, S. Mansfield, I. Stobert, IBM (United States); C. Sarma, Infineon Technologies (United States); G. Lembach, Advanced Micro Devices (United States); J. Liu, Chartered Semiconductor (United States); K. Herold, Infineon Technologies (United States)

727413 Integrating assist feature print fixing with OPC [7274-38]

L. D. Barnes, A. Poonawala, B. D. Painter, A. M. Jost, T. Takei, Y. Li, Synopsys, Inc. (United States)

727414 Double-patterning-friendly OPC [7274-39]

X. Li, G. Luk-Pat, Synopsys, Inc. (United States); C. Cork, Synopsys, Inc. (France); L. Barnes,

K. Lucas, Synopsys, Inc. (United States)

SESSION 10 OPTICAL PROXIMITY CORRECTIONS II

727415 Calibrating OPC model with full CD profile data for 2D and 3D patterns using scatterometry [7274-40]

A. D. Dave, Mentor Graphics Corp. (United States); O. Kritsun, Y. Deng, K. Yoshimoto,

GLOBALFOUNDRIES (United States); J. Li, J. Hu, Nanometrics, Inc. (United States)

727416 Impact of modelisation pixel size on OPC consistency [7274-41]

F. Foussadier, E. Yesilada, J.-C. Le Denmat, STMicroelectronics (France); Y. Troviller, CEA/Leti (France); V. Farys, F. Robert, G. Kerrien, C. Gardin, STMicroelectronics (France); L. Perraud, CEA/Leti (France); F. Vautrin, A. Villaret, C. Martinelli, J. Planchot, STMicroelectronics (France); J. L. Di-Maria, CEA/Leti (France); M. Saied, M. K. Top, STMicroelectronics (France)

727417 OPC simplification and mask cost reduction using regular design fabrics [7274-42]

T. Jhaveri, Carnegie Mellon Univ. (United States) and PDF Solutions (United States); I. Stobert,

L. Liebmann, IBM Microelectronics (United States); P. Karakatsanis, PDF Solutions (Germany); V. Rovner, A. Strojwas, L. Pileggi, Carnegie Mellon Univ. (United States) and PDF Solutions (United States)

727418 Resist development modeling for OPC accuracy improvement [7274-43] Y. Fan, L. Zavyalova, Y. Zhang, C. Zhang, K. Lucas, B. Falch, E. Croffie, J. Li, L. Melvin, B. Ward, Synopsys, Inc. (United States) 
727419 OPC for reduced process sensitivity in the double patterning flow [7274-44]

M. Gheith, L. Hong, J. Word, Mentor Graphics Corp. (United States)

7274 IA Through-focus pattern matching applied to double patterning [7274-45]

J. Rubinstein, A. R. Neureuther, Univ. of California, Berkeley (United States)

\section{SESSION 12 RESOLUTION ENHANCEMENT}

7274 1B A novel methodology for hybrid mask AF generation for 22 and 15nm technology [7274-46]

Y. Zou, L. Capodieci, C. Tabery, Advanced Micro Devices (United States)

7274 1C Pushing the limits of RET with different illumination optimization methods [7274-129]

A. D. Dave, Mentor Graphics Corp. (United States); R. Kim, Advanced Micro Devices, Inc. (United States)

7274 IE A computational technique to optimally design in-situ diffractive elements: applications to projection lithography at the resist resolution limit [7274-50]

G. R. Feijóo, Woods Hole Oceanographic Institution (United States); J. Tirapu-Azpiroz, IBM SRDC Microelectronics (United States); A. E. Rosenbluth, IBM Thomas J. Watson Research Ctr. (United States); A. A. Oberai, J. J. Mohan, Rensselaer Polytechnic Institute (United States); K. Tian, IBM SRDC Microelectronics, (United States); D. Melville, D. Gil, IBM Thomas J. Watson Research Ctr. (United States); K. Lai, IBM SRDC Microelectronics (United States)

7274 lF Feasibility of ultra-low k1 lithography for 28nm CMOS node [7274-51]

S. Mimotogi, K. Takahata, Toshiba Corp. Semiconductor Co. (Japan); T. Murakami, S. Nagahara, K. Takeda, NEC Electronics Corp. (Japan); M. Satake, Y. Kitamura, T. Ojima,

H. Fujise, Y. Seino, T. Ema, H. Yonemitsu, M. Takakuwa, S. Nakagawa, T. Kono, M. Asano, S. Kyoh, H. Harakawa, A. Nomachi, T. Ishida, S. Hasegawa, K. Miyashita, Toshiba Corp. Semiconductor Co. (Japan); M. Tominaga, NEC Electronics Corp. (Japan); S. Inove, Toshiba Corp. Semiconductor Co. (Japan)

\section{SESSION 13 PROCESS}

7274 1G Defectivity improvement by modified wafer edge treatment in immersion lithography [7274-103] M. Fujita, T. Tamura, N. Onoda, T. Uchiyama, NEC Electronics Corp. (Japan)

$72741 \mathrm{H} \quad$ Design of resist stacks with antireflection coatings from the viewpoint of focus budget [7274-53]

S. Nagai, K. Sato, Toshiba Corp. Semiconductor Co. (Japan)

SESSION 14 TOOLS I

$727411 \quad$ An innovative platform for high-throughput high-accuracy lithography using a single wafer stage [7274-54] Y. Shibazaki, H. Kohno, M. Hamatani, Nikon Corp. (Japan) 
$72741 \mathrm{~J} \quad$ Novel approaches to meet the requirements for double patterning [7274-55]

T. Ebihara, T. Yoshihara, H. Morohoshi, T. Makiyama, Y. Kawanobe, K. Tsujita, T. Kojima,

K. Takahashi, Canon, Inc. (Japan)

$72741 \mathrm{~K}$ Extending single-exposure patterning towards 38-nm half-pitch using 1.35 NA immersion [7274-56]

I. Bouchoms, A. Engelen, J. Mulkens, H. Boom, R. Moerman, P. Liebregts, R. de Graaf, M. van Veen, P. Thomassen, ASML Netherlands B.V. (Netherlands); W. Emer, Carl Zeiss SMT AG (Germany); F. Sperling, ASML Netherlands B.V. (Netherlands)

7274 IL Advanced aberration control in projection optics for double patterning [7274-57] T. Yoshihara, T. Sukegawa, N. Yabu, M. Kobayashi, T. Arai, T. Kitamura, A. Shigenobu, Y. Hasegawa, K. Takahashi, Canon, Inc. (Japan)

7274 1M Polarization aberration control for ArF projection lenses [7274-58]

T. Matsuyama, N. Kita, Nikon Corp. (Japan)

\section{SESSION 15 TOOLS II}

727410 Modeling laser bandwidth for OPC applications [7274-60]

C. Zuniga, K. Adam, M. Lam, Mentor Graphics Corp. (United States); I. Lalovic, Cymer, Inc. (United States); P. De Bisschop, IMEC (Belgium)

7274 IP Control and reduction of immersion defectivity for yield enhancement at high volume production [7274-61]

K. Nakano, R. Seki, T. Sekito, M. Yoshida, T. Fujiwara, Y. Iriuchijima, S. Owa, Nikon Corp. (Japan)

7274 1Q Imaging solutions for the 22nm node using 1.35NA [7274-62]

A. Engelen, M. Mulder, I. Bouchoms, ASML (Netherlands); S. Hansen, ASML US, Inc. (United States); A. Bouma, A. Ngai, M. van Veen, ASML (Netherlands); J. Zimmermann, Carl Zeiss SMT AG (Germany)

7274 IR Speckle in optical lithography and the influence on line width roughness [7274-63]

O. Noordman, A. Tychkov, J. Baselmans, ASML Netherlands B.V. (Netherlands);

J. Tsacoyeanes, G. Politi, ASML Wilton (United States); M. Patra, V. Blahnik, M. Maul, Carl Zeiss SMT AG (Germany)

7274 is Enabling the lithography roadmap: an immersion tool based on a novel stage positioning system [7274-64]

F. de Jong, B. van der Pasch, T. Castenmiller, B. Vleeming, R. Droste, F. van de Mast, ASML Netherlands B.V. (Netherlands) 


\section{Part Two}

POSTER SESSION: DOUBLE PATTERNING

7274 IU A CDU comparison of double patterning process options using Monte Carlo simulation [7274-66]

J. Hooge, Tokyo Electron America (United States); S. Hatakeyama, K. Nafus, Tokyo Electron Kyushu, Ltd. (Japan); S. Scheer, Tokyo Electron America (United States); P. Foubert, S. Cheng, P. Leray, IMEC (Belgium)

7274 IV Exploration of linear and non-linear double exposure techniques by simulation [7274-67] J. S. Petersen, R. T. Greenway, Petersen Advanced Lithography, Inc. (United States); T. Fühner, P. Evanschitzky, F. Shao, A. Erdmann, Fraunhofer Institute of Integrated Systems and Device Technology (Germany)

$72741 \mathrm{X}$ Integration of dry etching steps for double patterning and spacer patterning processes [7274-69]

S. Barnola, C. Lapeyre, I. Servin, C. Arvet, P. Maury, L. Mage, CEA/LETI-Minatec (France)

7274 IY Analysis of higher order pitch division for sub-32nm lithography [7274-70]

P. Xie, B. W. Smith, Rochester Institute of Technology (United States)

\section{POSTER SESSION: HIGH INDEX LITHOGRAPHY}

$727412 \quad 32 \mathrm{~nm}$ half pitch formation with high numerical aperture single exposure [7274-71] M. Jung, J.-M. Park, Hanyang Univ. (Korea, Republic of); M. Kim, S. Hong, J. Kim, Seoul National Univ. (Korea, Republic of); I.-H. Park, Univ. of Incheon (Korea, Republic of); H.-K. Oh, Hanyang Univ. (Korea, Republic of)

727420 High index $193 \mathrm{~nm}$ immersion lithography: the beginning or the end of the road [7274-72] P. A. Zimmerman, B. J. Rice, Intel Corp. (United States) and SEMATECH Inc. (United States); E. C. Piscani, SEMATECH Inc. (United States); V. Liberman, MIT Lincoln Lab. (United States)

$727421 \quad$ Birefringence issues with uniaxial crystals as last lens elements for high-index immersion lithography [7274-73]

J. H. Burnett, E. C. Benck, S. G. Kaplan, National Institute of Standards and Technology

(United States); G. Y. Sirat, Crystalith (Israel); C. Mack, Crystalith (United States)

\section{POSTER SESSION: MASKS}

$727422 \quad$ Multiple layer CD control treatment [7274-76]

A. Birnstein, C. Röpke, M. Sczyrba, AMTC GmbH \& Co. KG (Germany); R. Pforr, M. Hennig, Qimonda Dresden GmbH \& Co. OHG (Germany); G. Ben-Zvi, E. Graitzer, A. Cohen, Pixer Technology Ltd. (Israel)

727423 Smart data filtering for enhancement of model accuracy [7274-77]

S. Abdelwahed, Mentor Graphics Corp. (Egypt); J. H. Kang, J. Choi, J. D. Kim, H. Lee, S. Jun, Y. Kim, Dongbu HiTek Co., Ltd. (Korea, Republic of) 
727424 Analysis and modeling of photomask edge effects for 3D geometries and the effect on process window [7274-78]

M. A. Miller, A. R. Neureuther, Univ. of California, Berkeley (United States)

POSTER SESSION: OPTICAL PROXIMITY CORRECTIONS

727425 Transformation procedure from sparse OPC model to grid-based model [7274-79]

Q. Liu, Semiconductor Manufacturing International Corp. (China); L. Zhang, Mentor Graphics Corp. (China)

727426 Image-assistant OPC model calibration on 65nm node contact layer [7274-80]

Y. Y. Tsai, S. L. Tsai, F. LO, E. Yang, T. H. Yang, K. C. Chen, C.-Y. LU, Macronix International Co., Ltd. (Taiwan)

727427 Design driven test patterns for OPC models calibration [7274-82]

M. Al-Imam, Mentor Graphics Corp. (Egypt)

727428 Model-based retarget for 45nm node and beyond [7274-85]

E. Yang, C. H. Li, Semiconductor Manufacturing International Corp. (China); X. H. Kang, Mentor Graphics Corp. (China); E. Guo, Semiconductor Manufacturing International Corp. (China)

727429 Pattern matching assisted modeling test pattern generation [7274-87]

L. Hong, Q. Li, J. Rao, Mentor Graphics Corp. (United States)

7274 2A Model based mask process correction and verification for advanced process nodes [7274-89]

T. Lin, T. Donnelly, S. Schulze, Mentor Graphics Corp. (United States)

$72742 \mathrm{C}$ Novel OPC method to create sub $45 \mathrm{~nm}$ contact hole using design based metrology [7274-91]

D.-J. Lee, S. Oh, J. Park, J. Choi, J. Kim, C. Kim, D. Yim, Hynix Semiconductor, Inc. (Korea, Republic of)

7274 2D Abbe-PCA (Abbe-Hopkins): microlithography aerial image analytical compact kernel generation based on principle component analysis [7274-92]

M.-F. Tsai, S.-J. Chang, C. C. P. Chen, National Taiwan Univ. (Taiwan); L. S. Melvin III, Synopsys, Inc. (United States)

72742 F Automatic SRAF size optimization during OPC [7274-98]

S. Jayaram, J. Word, Mentor Graphics Corp. (United States)

7274 2G OPC segmentation: dilemma between degree-of-freedom and stability with some relieves [7274-99]

Y. P. Tang, J. H. Feng, M. H. Chih, C. K. Tsai, W. C. Huang, C. C. Kuo, R. G. Liu, H. T. Lin, Y. C. Ku, Taiwan Semiconductor Manufacturing Co. (Taiwan)

$72742 \mathrm{H} \quad$ Efficient hardware usage in the mask tapeout flow [7274-100]

M. Boman, T. Brist, Y. Wang, Synopsys, Inc. (United States) 
$727421 \quad$ Pre-OPC layout decomposition for improved image fidelity [7274-101]

S. Abdelwahed, Mentor Graphics Corp. (Egypt); R. Fathy, Mentor Graphics Corp. (United

States); J. H. Kang, J. D. Kim, Y. Kim, Dongbu HiTek Co., Ltd. (Korea, Republic of)

\section{POSTER SESSION: PROCESS}

7274 2J Next generation siloxane-based Bottom Anti-Reflective Coating (BARC) formulations with selective strip rates and required optical properties [7274-102]

S. Mukhopadhyay, J. Kennedy, Y. Pandey, P. Amin, J. Gill, Honeywell Electronic Materials (United States)

$72742 \mathrm{~K} \quad$ Thin hardmask patterning stacks for the 22-nm node [7274-104]

Z. Zhu, Brewer Science, Inc. (United States); E. Piscani, SEMATECH Inc. (United States);

Y. Wang, J. Macie, C. J. Neef, B. Smith, Brewer Science, Inc. (United States)

$72742 \mathrm{~L} \quad$ Arbitrary three-dimensional micro-fabrication by polymer grayscale lithography [7274-105] L. Jiang, P. Nath, Tuskegee Univ. (United States); N. S. Korivi, Lovisiana State Univ. (United States)

$72742 \mathrm{~N} \quad \mathbf{0 . 1 3 \mu \mathrm { m }} \mathrm{BiCMOS}$ emitter window lithography with KrF scanners [7274-107]

L.-H. Chou, N. S. Patel, P. M. McCarthy, National Semiconductor Corp. (United States)

727420 Patterning of SU-8 resist with digital micromirror device (DMD) maskless lithography [7274-109]

T. Wang, Univ. of Wisconsin, Madison (United States); M. Quaglio, F. Pirri, Polytechnic of Torino (Italy); Y.-C. Cheng, D. Busacker, Univ. of Wisconsin, Madison (United States);

F. Cerrina, Univ. of Wisconsin, Madison (United States) and Univ. of Boston (United States)

\section{POSTER SESSION: PROCESS CONTROL}

$72742 \mathrm{P} \quad$ Process transfer strategies between ASML immersion scanners [7274-113]

Y. He, Micron Technology, Inc. (United States); P. Engblom, ASML (United States); J. Zhou, Micron Technology, Inc. (United States); E. Janda, ASML (United States); A. Devilliers, Micron Technology, Inc. (United States); B. Geh, Carl Zeiss SMT AG (Germany); E. Byers, Micron Technology, Inc. (United States); J. Menger, Carl Zeiss SMT AG (Germany); S. Hansen, M. Dusa, ASML (United States)

$72742 \mathrm{3} \quad 32 \mathrm{~nm}$ node device laser-bandwidth OPE sensitivity and process matching [7274-115] K. Yoshimochi, T. Tamura, T. Kuribayashi, T. Uchiyama, NEC Electronics Corp. (Japan); N. Farrar, T. Oga, Cymer, Inc. (United States); J. Bonafede, Cymer Japan, Inc. (Japan)

$72742 S \quad$ New approach to determine best beam focus [7274-117] C. Zuniga, T. M. Tawfik, Mentor Graphics Corp. (United States)

$72742 \mathrm{~T} \quad$ High-order distortion effects induced by extreme off-axis illuminations at hyper NA lithography [7274-118]

P. Rigolli, G. Capetti, E. De Chiara, Numonyx (Italy); L. Amato, STMicroelectronics (Italy);

U. lessi, P. Canestrari, Numonyx (Italy); C. Llorens, ASML Rousset (France); S. Smit, ASML Netherlands B.V. (Netherlands); L. Brige, ASML Rousset (France); J. Plauth, ASML Italy srl (Italy) 
$72742 \mathrm{~V}$ Line end shortening and corner rounding for novel off-axis illumination source shapes [7274-124]

M. L. Ling, National Univ. of Singapore (Singapore); G. S. Chua, Q. Lin, Chartered Semiconductor Manufacturing, Ltd. (Singapore); C. J. Tay, C. Quan, National Univ. of Singapore (Singapore)

$72742 \mathrm{~W}$ The analysis of polarization characteristics on 40nm memory devices [7274-125] M. Yoo, C. Park, T. You, H. Yang, Hynix Semiconductor, Inc. (Korea, Republic of); Y.-H. Min, K.-Y. Park, ASML Korea (Korea, Republic of); D. Yim, S. Park, Hynix Semiconductor, Inc. (Korea, Republic of)

$72742 X \quad 22 \mathrm{~nm}$ technology node active layer patterning for planar transistor devices [7274-126] R. Kim, Advanced Micro Devices, Inc. (United States); S. Holmes, S. Halle, IBM Research (United States); V. Dai, Advanced Micro Devices, Inc. (United States); J. Meiring, IBM SRDC (United States); A. Dave, Mentor Graphics Corp. (United States); M. E. Colburn, IBM Research (United States); H. J. Levinson, Advanced Micro Devices, Inc. (United States)

$72742 Y \quad C$-quad polarized illumination for back end thin wire: moving beyond annular illumination regime [7274-128]

S. S. Mehta, Chartered Semiconductor Manufacturing, Ltd. (United States); H.-R. Lee, SAMSUNG Electronics Co., Ltd. (United States); B. Hamieh, STMicroelectronics N.V. (United States); C. Kallingal, I. Matthew, Advanced Micro Devices, Inc. (United States); R. Viswanathan, D. N. Dunn, IBM Semiconductor Research and Development Ctr. (United States)

\section{POSTER SESSION: SIMULATION}

$72742 Z \quad$ A proposed image intensity expressing local irradiance [7274-131] S. Nakao, I. Kanai, S. Maejima, M. Okuno, N. Tamada, J. Sakai, A. Imai, T. Hanawa, K. Suko, Renesas Technology (Japan)

727430 The divergence of image and resist process metrics [7274-132] J. J. Biafore, S. Kapasi, S. A. Robertson, M. D. Smith, KLA-Tencor (United States)

727431 Advanced model and fast algorithm for aerial image computation with well controlled accuracy [7274-133]

V. Manuylov, Robust Chip Inc. (United States)

727432 A novel fast 3D resist simulation method using Chebyshev expansion [7274-134] M. Takahashi, S. Tanaka, S. Mimotogi, S. Inove, Toshiba Corp. Semiconductor Co. (Japan)

727433 Modeling mask scattered field at oblique incidence (Best Student Paper Award) [7274-135] T. M. Tawfik, Mentor Graphics Corp. (Egypt); A. H. Morshed, D. Khalil, Ain Shams Univ. (Egypt)

727434 Partially coherent image computation using elementary functions [7274-136]

A. Smith, A. Burvall, C. Dainty, National Univ. of Ireland, Galway (Ireland) 
727435 Chemically amplified resist modeling in OPC [7274-137]

$X$. Zheng, J. Huang, Synopsys, Inc. (United States); F. Kuo, Synopsys, Inc. (Taiwan);

A. Kazarian, F. Chin, Y. Fan, Synopsys, Inc. (United States)

727436 High-speed microlithography aerial image contour generation without images [7274-138] S. Lin, C. C. P. Chen, National Taiwan Univ. (Taiwan); L. S. Melvin III, Synopsys, Inc. (United States)

\section{POSTER SESSION: SOURCE AND MASK OPTIMIZATION}

727437 PSM design for inverse lithography with partially coherent illumination [7274-139]

X. Ma, G. R. Arce, Univ. of Delaware (United States)

727439 Manufacturability of ILT patterns in low-NA 193nm environment [7274-141]

C. Lim, V. Temchenko, I. Meusel, D. Kaiser, J. Schneider, Infineon Technologies Dresden

GmbH and Co. OHG (Germany); M. Niehoff, Mentor Graphics Corp. (Germany)

7274 3A Inverse vs. traditional OPC for the 22nm node [7274-142]

J. Word, Y. Granik, M. Medvedeva, S. Rodin, Mentor Graphics Corp. (United States);

L. Capodieci, Y. Deng, J. Kye, C. Tabery, K. Yoshimoto, Y. Zou, Advanced Micro Devices

(United States); H. Diab, Mentor Graphics Corp. (Egypt); M. Gheith, Mentor Graphics Corp. (United States); M. Habib, Mentor Graphics Corp. (Egypt); C. Zhu, Mentor Graphics Corp.

(United States)

7274 3B Innovative pixel-inversion calculation for model-based sub-resolution assist features and optical proximity correction [7274-143]

J.-C. YU, P. YU, H.-Y. Chao, National Chiao-Tung Univ. (Taiwan)

7274 3D Source optimization for three-dimensional image designs through film stacks [7274-145]

D. O. S. Melville, A. E. Rosenbluth, IBM Thomas J. Watson Research Ctr. (United States);

K. Tian, IBM Semiconductor Research and Development Ctr. (United States); D. Goldfarb, IBM Thomas J. Watson Research Ctr. (United States); S. Harrer, M. Colburn, IBM Research at Albany NanoTech (United States)

\section{POSTER SESSION: SPACER-BASED PROCESSES}

7274 3E Pattern decomposition and process integration of self-aligned double patterning for 30nm node NAND FLASH process and beyond [7274-147]

Y.-S. Chang, M.-F. Tsai, C.-C. Lin, J.-C. Lai, Powerchip Semiconductor Corp. (Taiwan)

$72743 \mathrm{~F} \quad$ A manufacturing lithographic approach for high density MRAM device using KrF double mask patterning technique [7274-146]

D. Liu, T. Zhong, T. Torng, Magic Technologies (United States)

$72743 \mathrm{G}$ Alignment and overlay improvements for $3 \mathrm{x} \mathrm{nm}$ and beyond process with CVD sidewall spacer double patterning [7274-148]

H. Dai, C. Bencher, Y. Chen, S. Sun, X. Xu, C. Ngai, Applied Materials, Inc. (United States) 
$72743 \mathrm{H} \quad$ Birefringence simulations of annealed ingot of calcium fluoride single crystal: consideration of creep behavior of ingot during annealing process [7274-151]

N. Miyazaki, H. Ogino, Y. Kitamura, Kyoto Univ. (Japan); T. Mabuchi, T. Nawata, Tokuyama Corp. (Japan)

$727431 \quad$ Cost-effective shrink of semi-critical layers using the TWINSCAN XT:1000H NA $0.93 \mathrm{KrF}$ scanner [7274-152]

F. Bornebroek, M. de Wit, W. de Boeij, G. Dicker, ASML (Netherlands); J. Hong, ASML (Taiwan); A. Serebryakov, ASML (Netherlands)

7274 3J Optical performance of laser light source for ArF immersion double patterning lithography tool [7274-153]

K. Wakana, H. Tsushima, S. Matsumoto, M. Yoshino, T. Kumazaki, H. Watanabe, T. Ohta,

S. Tanaka, T. Suzuki, H. Nakarai, Y. Kawasuji, A. Kurosu, T. Matsunaga, J. Fujimoto,

H. Mizoguchi, Gigaphoton Inc. (Japan)

7274 3K True polarization characteristics of hyper-NA optics excluding impact of measurement system [7274-155]

T. Fujii, K. Muramatsu, Nikon Corp. (Japan); N. Matsuo, Nikon Systems, Inc. (Japan);

Y. Ohmura, Nikon Corp. (Japan); M. Sawada, Nikon Systems, Inc. (Japan)

7274 3 Reliability report of high power injection lock laser light source for double exposure and double patterning ArF immersion lithography [7274-156]

H. Tsushima, M. Yoshino, T. Ohta, T. Kumazaki, H. Watanabe, S. Matsumoto, H. Nakarai,

H. Umeda, Y. Kawasuji, T. Suzuki, S. Tanaka, A. Kurosu, T. Matsunaga, J. Fujimoto,

H. Mizoguchi, Gigaphoton Inc. (Japan)

7274 3M Immersion-cluster uptime enhancement technology toward high-volume manufacturing [7274-157]

R. Tanaka, T. Fujiwara, K. Nakano, Nikon Corp. (Japan); S. Wakamizu, H. Kyouda, Tokyo Electron Kyushu, Ltd. (Japan)

7274 3N Lithography line productivity impact using Cymer GLX technology [7274-158]

K. O'Brien, W. J. Dunstan, R. Jacques, D. Brown, Cymer, Inc. (United States)

727430 Enabling high volume manufacturing of double patterning immersion lithography with the XLR 600ix ArF light source [7274-159]

R. Rokitski, V. Fleurov, R. Bergstedt, H. Ye, R. Rafac, R. Jacques, F. Trintchouk, T. Ishihara,

R. Rao, T. Cacouris, D. Brown, W. Partlo, Cymer, Inc. (United States) 
Downloaded From: https://www.spiedigitallibrary.org/conference-proceedings-of-spie on 26 Apr 2023

Terms of Use: https://www.spiedigitallibrary.org/terms-of-use 


\title{
Symposium Committees
}

\author{
Symposium Chair
}

Christopher J. Progler, Photronics, Inc. (United States)

Symposium Cochair

Donis G. Flagello, Nikon Corporation of America (United States)

Technical Organizing Committees

Executive Committee

John A. Allgair, SEMATECH Inc. (United States) and Advanced Micro Devices, Inc. (United States)

Mircea V. Dusa, ASML MaskTools, Inc. (United States)

Donis G. Flagello, Nikon Corporation of America (United States)

Clifford L. Henderson, Georgia Institute of Technology (United States)

Bruno La Fontaine, Advanced Micro Devices, Inc. (United States)

Harry J. Levinson, Advanced Micro Devices, Inc. (United States)

Christopher J. Progler, Photronics, Inc. (United States)

Christopher J. Raymond, Nanometrics, Inc. (United States)

Michael L. Rieger, Synopsys, Inc. (United States)

Franklin M. Schellenberg, Mentor Graphics Corporation (United States)

Vivek K. Singh, Intel Corporation (United States)

Advisory Committee

Robert D. Allen, IBM Almaden Research Center (United States)

William H. Arnold, ASML US Inc. (United States)

Timothy A. Brunner, IBM Thomas J. Watson Research Center (United States)

Ralph R. Dammel, AZ Electronic Materials USA Corporation (United States)

Roxann L. Engelstad, University of Wisconsin, Madison (United

States)

Roderick R. Kunz, MIT Lincoln Laboratory (United States)

Harry J. Levinson, Advanced Micro Devices, Inc. (United States)

Burn Lin, Taiwan Semiconductor Manufacturing Company, Ltd. (Taiwan)

Chris A. Mack, LithoGuru.com (United States)

Victor Pol, Freescale Semiconductor, Inc. (United States) 
Michael T. Postek, National Institute of Standards and Technology (United States)

Luc Van den Hove, IMEC vzW (Belgium)

C. Grant Willson, The University of Texas at Austin (United States) Anthony Yen, Taiwan Semiconductor Manufacturing Company, Ltd. (Taiwan) 


\title{
Conference Committee
}

\author{
Conference Chair
}

Harry J. Levinson, Advanced Micro Devices, Inc. (United States)

Conference Cochair

Mircea V. Dusa, ASML MaskTools, Inc. (United States)

Program Committee

Pary Baluswamy, Micron Technology, Inc. (United States)

Willard E. Conley, Freescale Semiconductor, Inc. (United States)

Nigel R. Farrar, Cymer, Inc. (United States)

Roger H. French, DuPont Company (United States)

Tsai-Sheng Gau, Taiwan Semiconductor Manufacturing Company, Ltd. (Taiwan)

Tatsuhiko Higashiki, Toshiba Corporation (Japan)

Kurt R. Kimmel, Advanced Mask Technology Center GmbH \& Company KG (Germany)

Kafai Lai, IBM Microelectronics Division (United States)

SukJoo Lee, SAMSUNG Electronics Company, Ltd. (Korea, Republic of)

Wilhelm Maurer, Infineon Technologies AG (Germany)

Soichi Owa, Nikon Corporation (Japan)

Ken Ozawa, Sony Atsugi Technology Center (Japan)

Sam Sivakumar, Intel Corporation (United States)

Bruce W. Smith, Rochester Institute of Technology (United States)

Kazuhiro Takahashi, Canon, Inc. (Japan)

Geert Vandenberghe, IMEC (Belgium)

\section{Session Chairs}

1 Invited Session

Harry J. Levinson, Advanced Micro Devices, Inc. (United States)

Mircea V. Dusa, ASML MaskTools, Inc. (United States)

2 Resolution Enhancement

Kafai Lai, IBM Microelectronics Division (United States)

Willard E. Conley, Freescale Semiconductor, Inc. (United States)

3 Source and Mask Optimization

Tatsuhiko Higashiki, Toshiba Corporation (Japan)

Sam Sivakumar, Intel Corporation (United States) 
Kazuhiro Takahashi, Canon, Inc. (Japan)

Geert Vandenberghe, IMEC (Belgium)

5 Double Patterning I

Soichi Owa, Nikon Corporation (Japan)

Ken Ozawa, Sony Corporation (Japan)

6 Double Patterning II

Geert Vandenberghe, IMEC (Belgium)

Pary Baluswamy, Micron Technology, Inc. (United States)

$7 \quad$ Tools Related Process Control I

Kazuhiro Takahashi, Canon, Inc. (Japan)

Tsai-Sheng Gau, Taiwan Semiconductor Manufacturing Company, Ltd. (Taiwan)

8 Tools Related Process Control II

Nigel R. Farrar, Cymer, Inc. (United States)

Kurt R. Kimmel, Advanced Mask Technology Center GmbH \& Company KG (Germany)

9 Optical Proximity Corrections I

Willard E. Conley, Freescale Semiconductor, Inc. (United States)

Geert Vandenberghe, IMEC (Belgium)

10 Optical Proximity Corrections II

Sam Sivakumar, Intel Corporation (United States)

Kafai Lai, IBM Corporation (United States)

11 Optical Proximity Corrections III

Ken Ozawa, Sony Atsugi Technology Center (Japan)

Kurt R. Kimmel, Advanced Mask Technology Center GmbH \& Company KG (Germany)

12 Resolution Enhancement

Bruce W. Smith, Rochester Institute of Technology (United States)

Tsai-Sheng Gau, Taiwan Semiconductor Manufacturing Company, Ltd. (Taiwan)

13 Process

Ken Ozawa, Sony Atsugi Technology Center (Japan)

Kurt R. Kimmel, Advanced Mask Technology Center GmbH \& Company KG (Germany) 
14 Tools I

Nigel R. Farrar, Cymer, Inc. (United States)

Tatsuhiko Higashiki, Toshiba Corporation (Japan)

15 Tools II

Bruce W. Smith, Rochester Institute of Technology (United States)

Pary Baluswamy, Micron Technology, Inc. (United States) 
Downloaded From: https://www.spiedigitallibrary.org/conference-proceedings-of-spie on 26 Apr 2023

Terms of Use: https://www.spiedigitallibrary.org/terms-of-use 